\title{
Influences du degré d'harmonie des cinétiques de dégradation des fractions glucidiques et azotées des aliments concentrés sur les fermentations en Rusitec
}

\author{
S Giger-Reverdin, LS Munoz, D Sauvant, EM Leboute, P Chapoutot
}

INRA de I'INA-PG, station de Nutrition et Alimentation, 16, rue Claude-Bernard 75005 Paris, France

\begin{abstract}
Summary - In vitro study on the harmonization of degradation kinetics of the carbohydrate and nitrogen fractions of concentrates. Binary blends of ingredients of different composition and ruminal degradation rate (barley, corn, lupin, glutenmeal) were studied in a RUSITEC. There were positive effects of association on degradation and fermentation. However, the results obtained do not allow a definite conclusion to be made on the possibility of harmonizing the degradation kinetics of nitrogen and carbohydrates.
\end{abstract}

L'influence du degré d'harmonisation des cinétiques de dégradation des fractions glucidiques et azotées des aliments concentrés sur les fermentations ruminales demande à être précisée. Elle a été étudiée en biofermenteur Rusitec suite à l'étude réalisée in situ par Chapoutot et al (1990).

Matériel et méthodes - Quatre matières premières ont été choisies pour leur vitesse de dégradation ruminale rapide (orge- 0 , lupin- $L$ ) ou lente (maïs- $M$, glutenmeal- $G$ ), ainsi que pour leur richesse en glucides $(O, M)$ ou en protéines (L,G). Elles ont été combinées au sein de 4 mélanges "harmonieux" (OL,MG) ou non (OG,ML) incluant $60 \%$ de la source glucidique $(O, M)$ et $40 \%$ de la source azotée (L,G), et présentant respectivement des teneurs en matières azotées totales de $25,9,29,7,29,3,26,8 \%$ MS.

La méthodologie appliquée avec le Rusitec a été décrite par Durand et al (1988). Les aliments ont été broyés à la grille de $1 \mathrm{~mm}$ et introduits à raison de $13,5 \mathrm{~g} \mathrm{MS}$ par sachet. Les mesures ont été effectuées pendant $4 \mathrm{j}$ après $7 \mathrm{j}$ de prépériode.

Résultats et discussion - Le tableau I présente les principaux résultats obtenus. Les pourcentages de dégradation de la MS en 24 ou $48 \mathrm{~h}$ sont largement inférieurs à ce qui avait été obtenu in situ (Chapoutot et al, 1990). Les différences les plus impor- tantes à $24 \mathrm{~h}$ concernent $\mathrm{O}$ et $\mathrm{M}$, vraisemblablement en raison de la carence azotée des biofermenteurs correspondants. La dégradation de la MS en $24 \mathrm{~h}$ des 4 aliments composés est statistiquement supérieure à celle calculée à partir de celles des ingrédients et de leur pourcentage d'incorporation, ce qui traduit une interaction digestive positive. II en est de même pour la dégradation de la $M S$ en $48 \mathrm{~h}$ des mélanges à base de maïs ( $M G$ et $M L$ ).

Les hiérarchies entre les niveaux de dégradation, les productions de gaz et d'AGV des aliments sont assez cohérentes. Cependant, par rapport à son niveau de dégradation, $L$ apparaît comme un faible producteur de gaz et d'AGV, alors que la tendance est inverse pour $O$. La production d'NH $\mathrm{NH}_{3}$ est largement expliquée par la quantité d'azote dégradée. Les mélanges sont plus dégradés et fermentés et présentent des concentrations en AGV statistiquement plus élevées et une ammoniogenèse significativement plus faible que ne le laisseraient prévoir les résultats des ingrédients. La nature des aliments a peu d'influence sur le profil en AGV des effluents, puisque seules quelques différences significatives apparaissent sur l'acide propionique. Mais aucune interac- 
tion n'a été mise en évidence sur les pourcentages molaires en AGV.

La connaissance des cinétiques de dégradation in sacco des constituants analytiques de ces aliments (Chapoutot et al, 1990 et non publié) a permis, en se plaçant à même niveau de dégradabilité de la MS, de calculer les quantités de matière organique (MO) dégradée et d'azote théoriquement utilisé par les micro-organismes $(\mathrm{Nm})$, compte tenu de la fraction retrouvée sous forme d'NH $\mathrm{N}_{3}$. Le rapport $\mathrm{gNm} / \mathrm{kg} \mathrm{MO}$ dégradée est médiocre pour $0(17,3)$ et $M$ $(16,1)$, ce qui traduit peut-être une faible croissance microbienne causée par des phénomènes de découplage fermentaire. Ce rapport est compris entre 32,5 et 37,2 pour les aliments L, G, OL, OG et MG, il est sensiblement plus élevé $(45,3)$ pour l'aliment ML. Compte tenu du principe de la conservation des éléments en Rusitec (Guy et al, 1988), les différences ont été calculées entre, d'une part, les flux de $C$ issus de la dégradation des glucides, des apports salivaires et des acides aminés dégradés en $\mathrm{NH}_{3}$ et, d'autre part, les flux de $C$ retrouvés sous forme de gaz et d'AGV (tableau I). Les rapports $\mathrm{C} / \mathrm{N}$ utilisés sont faibles pour OL $(3,30)$, OG $(3,34)$ et MG $(3,42)$ et très faibles pour ML $(1,90)$ en comparaison de $L(5,67)$ et surtout $G$ $(8,69), O(11,33)$ et $M(19,51)$. Ces valeurs sont à rapprocher de celles des microorganismes $(5,5$ à 7,5$)$ pour estimer l'élément susceptible d'être le plus limitant de leur croissance.

En conclusion, les mélanges, en particulier ML, semblent aboutir à une meilleure efficacité de l'utilisation des fractions carbonées et azotées. Cependant les résultats obtenus ne permettent pas de conclure précisément sur l'opportunité d'harmoniser les cinétiques de dégradation des glucides et de l'azote.

Chapoutot $P$, Giger $S$, Sauvant D, Jeantet $S$ (1990) Reprod Nutr Dev 52, 169s-170s

Durand M, Dumay C, Beaumatin P, Morel AT (1988) Anim Fd Sci Techn 21, 197-204

Guy C, Jouany JP, Gros JB (1988) Reprod Nutr Dev 28, 145-146

Tableau I. Résultats des incubations des aliments en Rusitec ${ }^{1}$.

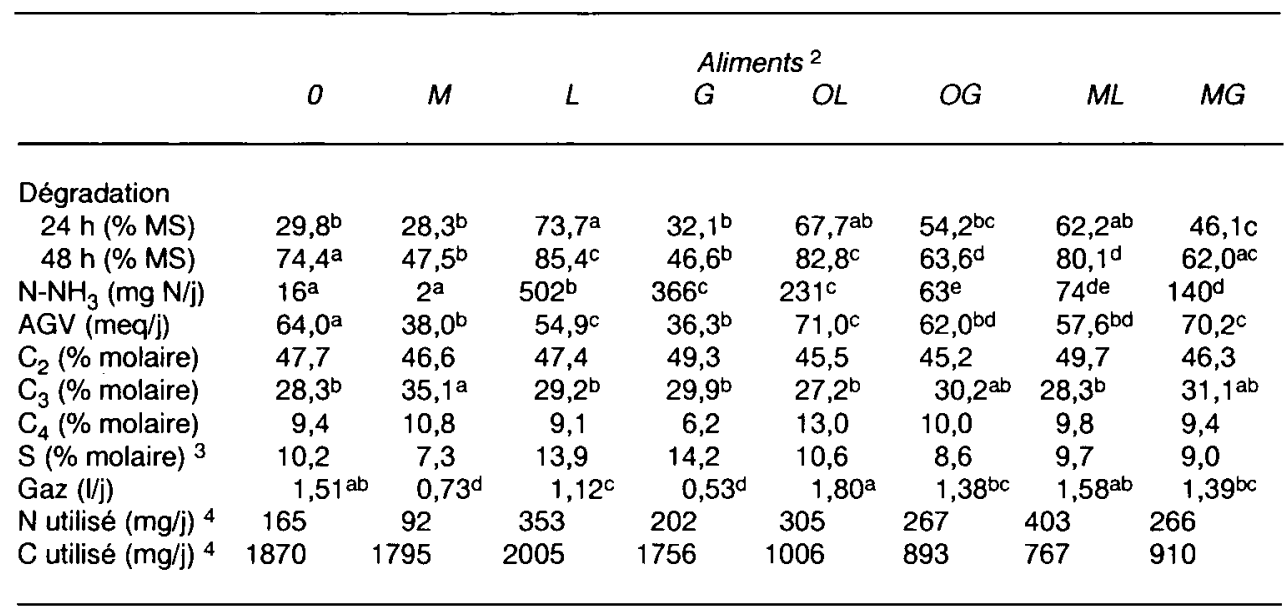

\footnotetext{
${ }^{1}$ Des indices de lettres differents séparent les effets significatifs $(P<0,05) ;{ }^{2} \mathrm{O}:$ orge, $\mathrm{M}:$ maïs, $\mathrm{L}:$ lupin, $\mathrm{G}:$ glutenmeal; ${ }^{3} \mathrm{~S}=$ iso $\mathrm{C}_{4}+$ iso $\mathrm{C}_{5}+\mathrm{C}_{5}+\mathrm{C}_{6}:{ }^{4}$ valeurs calculées par différence entre les apports et les quantités retrouvees en AGV, en gaz ou $\mathrm{NH}_{3}$.
} 